JOURNAL OF

SYMPLECTIC GEOMETRY

Volume 8, Number 4, 359-380, 2010

\title{
SYMMETRY OF A SYMPLECTIC TORIC MANIFOLD
}

\author{
MiKiYA MASUdA
}

\begin{abstract}
The action of a torus group $T$ on a symplectic toric manifold $(M, \omega)$ often extends to an effective action of a (non-abelian) compact Lie group $G$. We may think of $T$ and $G$ as compact Lie subgroups of the symplectomorphism group $\operatorname{Symp}(M, \omega)$ of $(M, \omega)$. On the other hand, $(M, \omega)$ is determined by the associated moment polytope $P$ by the result of Delzant [4]. Therefore, the group $G$ should be estimated in terms of $P$ or we may say that a maximal compact Lie subgroup of $\operatorname{Symp}(M, \omega)$ containing the torus $T$ should be described in terms of $P$. In this paper, we introduce a root system $R(P)$ associated to $P$ and prove that any irreducible subsystem of $R(P)$ is of type $A$ and the root system $\Delta(G)$ of the group $G$ is a subsystem of $R(P)$ (so that $R(P)$ gives an upper bound for the identity component of $G$ and any irreducible factor of $\Delta(G)$ is of type $A)$. We also introduce a homomorphism $\mathcal{D}$ from the normalizer $N_{G}(T)$ of $T$ in $G$ to an automorphism group $\operatorname{Aut}(P)$ of $P$, which detects the connected components of $G$. Finally, we find a maximal compact Lie subgroup $G_{\max }$ of $\operatorname{Symp}(M, \omega)$ containing the torus $T$ such that $\Delta\left(G_{\max }\right)=R(P)$ and $\mathcal{D}$ is onto.
\end{abstract}

\section{Introduction}

A symplectic toric manifold is a compact connected symplectic manifold $(M, \omega)$ with an effective Hamiltonian action of a torus group $T$ of half the dimension of the manifold $M$. Delzant [4] proves that $M$ is equivariantly diffeomorphic to a smooth projective toric variety with the restricted $T$-action. Moreover he classifies symplectic toric manifolds by showing that the correspondence from symplectic toric manifolds modulo equivalence to their moment polytopes is one-to-one. Therefore, all geometrical information on $(M, \omega)$ is encoded in the moment polytope $P$ associated with $(M, \omega)$.

The $T$-action on $(M, \omega)$ often extends to an effective action of a (nonabelian) compact Lie group $G$. We may think of $T$ and $G$ as compact Lie subgroups of the symplectomorphism group $\operatorname{Symp}(M, \omega)$ of $(M, \omega)$. Since 
the $T$-fixed point set in $M$ is non-empty and $\operatorname{dim} T=\frac{1}{2} \operatorname{dim} M$, there is no torus subgroup of $\operatorname{Symp}(M, \omega)$ containing $T$ properly. This means that $T$ is a maximal torus of $G$.

In this paper, we introduce a root system $R(P)$ associated to the moment polytope $P$ and a homomorphism

$$
\mathcal{D}: N_{G}(T) \rightarrow \operatorname{Aut}(P),
$$

where $N_{G}(T)$ denotes the normalizer of $T$ in $G$ and $\operatorname{Aut}(P)$ denotes an automorphism group of $P$. It turns out that the root system $R(P)$ gives information on the identity component $G^{0}$ of $G$ and the homomorphism $\mathcal{D}$ induces an injective homomorphism

$$
G / G^{0} \cong N_{G}(T) / N_{G^{0}}(T) \rightarrow \operatorname{Aut}(P) / \mathcal{D}\left(N_{G^{0}}(T)\right),
$$

so that $\mathcal{D}$ detects the connected components of $G$. Here is a summary of our results.

Theorem 1.1. Let $(M, \omega)$ be a symplectic toric manifold with a Hamiltonian action of a torus $T$ of half the dimension of the dimension of $M$ and let $P$ be the associated moment polytope. Then the following hold:

(1) Any irreducible subsystem of $R(P)$ is of type $A$.

(2) If $G$ is a compact Lie subgroup of $\operatorname{Symp}(M, \omega)$ containing the torus $T$, then the root system $\Delta(G)$ of $G$ is a subsystem of $R(P)$, so that any irreducible factor of $\Delta(G)$ is of type $A$ by (1) above.

(3) Let $G$ be as in (2) above. If $\Delta(G)=R(P)$ and the homomorphism $\mathcal{D}$ in (1.1) is surjective, then $G$ is maximal among compact Lie subgroups of $\operatorname{Symp}(M, \omega)$, i.e., $G$ is not properly contained in a compact Lie subgroup of $\operatorname{Symp}(M, \omega)$.

(4) There exists a compact Lie subgroup $G_{\max }$ of $\operatorname{Symp}(M, \omega)$ containing the torus $T$ such that the assumption in (3) above is satisfied.

Remark 1.1. By (3) above, the group $G_{\max }$ in (4) above, which contains the torus $T$, is maximal among compact Lie subgroups of $\operatorname{Symp}(M, \omega)$. However, the author does not know whether any maximal compact Lie subgroup of $\operatorname{Symp}(M, \omega)$ containing $T$ is conjugate to $G_{\max }$ in $\operatorname{Symp}(M, \omega)$, where the torus $T$ is fixed. Related to this question, it is proved in [8] that when $\operatorname{dim} M=4$, the number of conjugacy classes of 2-dimensional tori in $\operatorname{Symp}(M, \omega)$ is finite.

Our work is motivated by the work of Demazure [5] (see also [3] or [11, Section 3.5]). He introduces a root system $R(\Delta)$ for a complete non-singular fan $\Delta$ and proves that it agrees with the root system of the automorphism group $\operatorname{Aut}(X(\Delta))$ of the compact smooth toric variety $X(\Delta)$ associated with $\Delta$, where $\operatorname{Aut}(X(\Delta))$ is known to be an algebraic group, and that $R_{s}(\Delta):=R(\Delta) \cap(-R(\Delta))$ is the root system of the reductive (or semisimple) 
part of $\operatorname{Aut}(X(\Delta))$. The symplectic toric manifold $(M, \omega)$ is equivariantly diffeomorphic to a smooth projective toric variety $X$ with the restricted $T$-action as mentioned before and the fan $\Delta_{X}$ of the $X$ is the so-called normal fan derived from the moment polytope $P$ associated with $(M, \omega)$, where normal vectors $v_{i}$ 's to facets of $P$ are edge vectors in the fan $\Delta_{X}$. One sees that our root system $R(P)$ agrees with $R_{s}\left(\Delta_{X}\right)$. Demazure also describes the connected components of Aut $(X(\Delta))$ in terms of the automorphism group $\operatorname{Aut}(\Delta)$ of the fan $\Delta$. The automorphism group $\operatorname{Aut}(P)$ of $P$ can be regarded as a counterpart to $\operatorname{Aut}(\Delta)$; in fact, $\operatorname{Aut}(P)$ can be regarded as a subgroup of $\operatorname{Aut}(\Delta)$. We remark that the root systems $R(P)$ and $R(\Delta)$ depend only on the vectors $v_{i}$ 's but $\operatorname{Aut}(P)$ and $\operatorname{Aut}(\Delta)$ are not determined by the vectors.

This paper is organized as follows. In Section 2 we review an explicit construction (called the Delzant construction in [6]) of a symplectic toric manifold $(M, \omega)$ with moment polytope $P$. In Section 3 we rewrite the construction in terms of equivariant (co)homology and also recall some facts on the equivariant cohomology of $M$. In Section 4 we make some observations on roots of a compact Lie subgroup $G$ of $\operatorname{Symp}(M, \omega)$ containing the torus $T$. Based on the observations, we introduce the root system $R(P)$ and prove the assertions (1) and (2) in Theorem 1.1 (see Proposition 5.1, Theorem 5.1 and Corollary 5.1). In Section 6 we find a connected compact Lie subgroup $G$ of $\operatorname{Symp}(M, \omega)$, which attains the equality $\Delta(G)=R(P)$. In Section 7 we introduce the homomorphism $\mathcal{D}$ in (1.1) for an arbitrary subgroup $G$ of $\operatorname{Symp}(M, \omega)$ containing $T$ and observe that $\mathcal{D}$ detects the connected components of $G$ when $G$ is a compact Lie group. The assertions (3) and (4) in Theorem 1.1 are proved in Section 8 (see Theorem 8.1).

Throughout this paper, $(M, \omega)$ will denote a symplectic toric manifold with moment polytope $P$, where a Hamiltonian $T$-action on $(M, \omega)$ is incorporated, although it is often not mentioned explicitly. The argument developed in this paper works for torus manifolds introduced in [7] with some modification. We will discuss this in a forthcoming paper.

\section{Delzant construction}

By the result of Delzant mentioned in the Introduction, a symplectic toric manifold $(M, \omega)$ is determined by the associated moment polytope $P$ and is explicitly constructed from $P$. We will review the construction in this section. The details can be found in $[\mathbf{6}]$.

Let $\mu: M \rightarrow \mathfrak{t}^{*}$ be a moment map associated with $(M, \omega)$ so that $\mu(M)=$ $P$, where $\mathfrak{t}^{*}$ is the dual of the Lie algebra $\mathfrak{t}$ of $T$. The moment map $\mu$ is uniquely determined by $(M, \omega)$ up to parallel translations in $\mathfrak{t}^{*}$. We identify $\mathfrak{t}$ with $\mathbb{R}^{n}$ and $\mathfrak{t}^{*}$ with $\left(\mathbb{R}^{n}\right)^{*}$ and express

$$
P=\left\{u \in\left(\mathbb{R}^{n}\right)^{*} \mid\left\langle u, v_{i}\right\rangle \geq a_{i} \quad(i=1, \ldots, m)\right\}
$$


where $v_{i} \in \mathbb{Z}^{n}$ is primitive, $\langle$,$\rangle is a natural pairing (i.e., evaluation) and$ $a_{i} \in \mathbb{R}$. Without loss of generality, we may assume that there is no redundant inequality in (2.1) so that the intersection of $P$ with the hyperplane defined by $\left\langle u, v_{i}\right\rangle=a_{i}$ is a facet (i.e., codimension 1 face) of $P$ for each $i$, which we denote by $P_{i}$. So there are exactly $m$ facets in $P$. The moment polytope $P$ is non-singular, which means that $P$ is simple and whenever $n$ facets of $P$ meet at a vertex, the $n$ vectors $v_{i}$ 's normal to the $n$ facets form a basis of $\mathbb{Z}^{n}$. A non-singular polytope is called a Delzant polytope in $[6]$.

Let $e_{1}, \ldots, e_{m}$ be the standard basis of $\mathbb{Z}^{m}$ and consider the linear map $\pi_{*}: \mathbb{Z}^{m} \rightarrow \mathbb{Z}^{n}$ sending $e_{i}$ to $v_{i}$ for $i=1, \ldots, m$. Since $P$ is non-singular, $\pi_{*}$ is surjective and we have an exact sequence

$$
0 \rightarrow \operatorname{Ker} \pi_{*} \stackrel{\iota_{*}}{\longrightarrow} \mathbb{Z}^{m} \stackrel{\pi_{*}}{\longrightarrow} \mathbb{Z}^{n} \rightarrow 0
$$

where $\iota_{*}$ is the inclusion. Taking the dual of this sequence, we obtain an exact sequence

$$
0 \leftarrow\left(\operatorname{Ker} \pi_{*}\right)^{*} \stackrel{\iota^{*}}{\longleftarrow}\left(\mathbb{Z}^{m}\right)^{*} \stackrel{\pi^{*}}{\longleftarrow}\left(\mathbb{Z}^{n}\right)^{*} \leftarrow 0
$$

and one easily sees that

$$
\pi^{*}(u)=\sum_{i=1}^{m}\left\langle u, v_{i}\right\rangle e_{i}^{*} \quad \text { for any } u \in\left(\mathbb{Z}^{n}\right)^{*},
$$

where $e_{1}^{*}, \ldots, e_{m}^{*}$ denote the dual basis of $e_{1}, \ldots, e_{m}$.

The map $\pi_{*}$ (resp. $\pi^{*}$ ) extends to a linear map from $\mathbb{R}^{m}$ onto $\mathbb{R}^{n}$ (resp. from $\left(\mathbb{R}^{n}\right)^{*}$ to $\left.\left(\mathbb{R}^{m}\right)^{*}\right)$ and we use the same notation for the extended map. We define $\pi_{a}^{*}:\left(\mathbb{R}^{n}\right)^{*} \rightarrow\left(\mathbb{R}^{m}\right)^{*}$ by

$$
\pi_{a}^{*}(u):=\pi^{*}(u)-\sum_{i=1}^{m} a_{i} e_{i}^{*}=\sum_{i=1}^{m}\left(\left\langle u, v_{i}\right\rangle-a_{i}\right) e_{i}^{*} .
$$

The map $\pi_{a}^{*}$ embeds $P$ into the positive orthant of $\left(\mathbb{R}^{m}\right)^{*}$. The fiber product of $\pi_{a}^{*}$ and the (moment) map

$$
\Phi: \mathbb{C}^{m} \rightarrow\left(\mathbb{R}^{m}\right)^{*}
$$

sending $z=\left(z_{1}, \ldots, z_{m}\right)$ to $\frac{1}{2} \sum_{i=1}^{m}\left|z_{i}\right|^{2} e_{i}^{*}$ is

$$
\left\{(z, u) \in \mathbb{C}^{m} \times\left.\left(\mathbb{R}^{n}\right)^{*}\left|\frac{1}{2} \sum_{i=1}^{m}\right| z_{i}\right|^{2} e_{i}^{*}=\pi_{a}^{*}(u)\right\} .
$$

Since $\pi_{a}^{*}$ is injective and $\iota^{*}\left(\pi_{a}^{*}(u)\right)=-\sum_{i=1}^{m} a_{i} \iota^{*}\left(e_{i}^{*}\right)$ for any $u$, the first projection from $\mathbb{C}^{m} \times\left(\mathbb{R}^{n}\right)^{*}$ onto $\mathbb{C}^{m}$ maps the fiber product (2.6) diffeomorphically onto

$$
\mathcal{Z}_{P}:=\left\{z \in \mathbb{C}^{m} \mid \sum_{i=1}^{m}\left(\frac{1}{2}\left|z_{i}\right|^{2}+a_{i}\right) \iota^{*}\left(e_{i}^{*}\right)=0\right\} .
$$


Note that

$$
\mathcal{Z}_{P}=\Phi^{-1}\left(\pi_{a}^{*}(P)\right)
$$

Remark 2.1. The manifold $\mathcal{Z}_{P}$ is called the moment-angle manifold of $P$ and its topology is intensively studied in [2]. It is also studied in [1] from the viewpoint of real algebraic geometry.

We identify $\mathbb{R} / \mathbb{Z}$ with the unit circle $S^{1}$ of the complex numbers $\mathbb{C}$ through the exponential map $x \rightarrow \exp (2 \pi \sqrt{-1} x)$ and set

$$
T:=\left(S^{1}\right)^{n} \text {. }
$$

The map $\pi_{*}$ in (2.2) induces an epimorphism

$$
\mathcal{V}:\left(S^{1}\right)^{m} \rightarrow T
$$

and we make an identification

$$
\left(S^{1}\right)^{m} / \operatorname{Ker} \mathcal{V}=T
$$

through the map $\mathcal{V}$. An element $c=\left(c_{1}, \ldots, c_{n}\right) \in \mathbb{Z}^{n}$ defines a homomorphism

$$
\lambda_{c}: S^{1} \rightarrow T
$$

sending $g$ to $\left(g^{c_{1}}, \ldots, g^{c_{n}}\right)$ and we note that

$$
\mathcal{V}\left(g_{1}, \ldots, g_{m}\right)=\prod_{i=1}^{m} \lambda_{v_{i}}\left(g_{i}\right) \quad \text { for }\left(g_{1}, \ldots, g_{m}\right) \in\left(S^{1}\right)^{m} .
$$

An element $b=\left(b_{1}, \ldots, b_{n}\right)$ in $\mathbb{Z}^{n}$ also defines a homomorphism

$$
\chi^{b}: T \rightarrow S^{1}
$$

sending $\left(h_{1}, \ldots, h_{n}\right)$ to $\prod_{i=1}^{n} h_{i}^{b_{i}}$. Then we have

$$
\left(\chi^{b} \circ \lambda_{c}\right)(g)=g^{\langle b, c\rangle} \text { for } g \in S^{1}
$$

where $\langle b, c\rangle=\sum_{i=1}^{n} b_{i} c_{i}$. Since the intersection of the kernels of $\chi^{b}: T \rightarrow S^{1}$ for all $b$ is trivial, it follows from (2.11) and (2.12) that

$$
\operatorname{Ker} \mathcal{V}=\left\{\left(g_{1}, \ldots, g_{m}\right) \in\left(S^{1}\right)^{m} \mid \prod_{i=1}^{m} g_{i}^{\left\langle u, v_{i}\right\rangle}=1 \text { for } \forall u \in \mathbb{Z}^{n}\right\} \text {. }
$$

Remark 2.2. The elements $b$ and $u$ above are taken from $\mathbb{Z}^{n}$ but we will see that it would be better to regard them as elements of $\left(\mathbb{Z}^{n}\right)^{*}$ through the product $\langle$,$\rangle .$

The group $\left(S^{1}\right)^{m}$ acts on $\mathbb{C}^{m}$ by componentwise multiplication and this action leaves $\mathcal{Z}_{P}$ invariant. The map $\Phi$ in (2.5) induces a homeomorphism from the quotient $\mathbb{C}^{m} /\left(S^{1}\right)^{m}$ onto the positive orthant of $\left(\mathbb{R}^{m}\right)^{*}$ and $\Phi\left(\mathcal{Z}_{P}\right)=\pi_{a}^{*}(P)$. The action of $\left(S^{1}\right)^{m}$ restricted to Ker $\mathcal{V}$ is free on $\mathcal{Z}_{P}$ and 
the quotient $\mathcal{Z}_{P} / \operatorname{Ker} \mathcal{V}$ is known to be isomorphic to the given $M$. The standard symplectic form

$$
\omega_{0}:=\frac{\sqrt{-1}}{2} \sum_{i=1}^{m} d z_{i} \wedge d \bar{z}_{i}
$$

on $\mathbb{C}^{m}$ is invariant under the linear action of the unitary group $U(m)$. The form $\omega_{0}$ descends to the given $\omega$ on $M$. In fact, if

$$
q: \mathcal{Z}_{P} \rightarrow M=\mathcal{Z}_{P} / \operatorname{Ker} \mathcal{V}
$$

denotes the quotient map, then $\omega$ satisfies

$$
\omega_{0} \mid \mathcal{Z}_{P}=q^{*}(\omega)
$$

and is uniquely determined by this identity, where the left-hand side denotes the restriction of $\omega_{0}$ to $\mathcal{Z}_{P}$. The action of $\left(S^{1}\right)^{m}$ on $\mathcal{Z}_{P}$ induces an action of $T=\left(S^{1}\right)^{m} / \operatorname{Ker} \mathcal{V}$ on $M=\mathcal{Z}_{P} / \operatorname{Ker} \mathcal{V}$ and this $T$-action on $M$ preserves the symplectic form $\omega$.

As remarked before, the equation $\left\langle u, v_{i}\right\rangle=a_{i}$ defines the facet $P_{i}$ of $P$ for each $i=1, \ldots, m$, and

$$
\mathcal{Z}_{P_{i}}:=\Phi^{-1}\left(\pi_{a}^{*}\left(P_{i}\right)\right) \quad \text { and } \quad M_{i}:=q\left(\mathcal{Z}_{P_{i}}\right)
$$

are, respectively, closed smooth submanifolds of $\mathcal{Z}_{P}$ and $M$ of real codimension 2. We call $M_{i}$ 's the characteristic submanifolds of $M$. We see from (2.7) or (2.8) that

$$
\mathcal{Z}_{P_{i}}=\mathcal{Z}_{P} \cap\left\{z_{i}=0\right\}
$$

and hence it follows from (2.10) and (2.11) that

Lemma 2.1. For each $i$ the characteristic submanifold $M_{i}$ is fixed pointwise by the $S^{1}$-subgroup $\lambda_{v_{i}}\left(S^{1}\right)$ of $T$.

\section{Equivariant cohomology}

It is more convenient and natural to interpret the Delzant construction in terms of equivariant (co)homology. We will discuss it and also recall some facts on equivariant cohomology in this section. Recall that the equivariant homology and cohomology of a space $X$ with an action of the torus $T$ are, respectively, defined as

$$
H_{*}^{T}(X):=H_{*}\left(E T \times_{T} X\right) \quad \text { and } \quad H_{T}^{*}(X):=H^{*}\left(E T \times_{T} X\right),
$$

where $E T \rightarrow B T=E T / T$ is a universal principal $T$-bundle and $E T \times_{T} X$ is the quotient of $E T \times X$ by the $T$-action given by

$$
t(e, x)=\left(e t^{-1}, t x\right) \quad \text { for }(e, x) \in E T \times X \text { and } t \in T .
$$


Let $(M, \omega)$ be a symplectic toric manifold and let $M_{i}$ 's $(i=1, \ldots, m)$ be the characteristic submanifolds of $M$. Since the $\omega$ restricted to $M_{i}$ is again a symplectic form, the form $\omega$ and its restriction to $M_{i}$ define orientations on $M$ and $M_{i}$. Since $M$ and $M_{i}$ are oriented and the inclusion map from $M_{i}$ to $M$ is equivariant, it defines an equivariant Gysin homomorphism

$$
H_{T}^{*}\left(M_{i}\right) \rightarrow H_{T}^{*+2}(M),
$$

which raises the cohomological degree by 2 because the codimension of $M_{i}$ in $M$ is 2 . We denote by $\tau_{i}$ the image of the unit element $1 \in H_{T}^{0}\left(M_{i}\right)$ by the equivariant Gysin homomorphism. The cohomological degree of $\tau_{i}$ is 2 . We may think of $\tau_{i}$ as the Poincaré dual of the cycle $M_{i}$ in the equivariant setting. Since a cup product $\prod_{i \in I} \tau_{i}$ for $I \subset[m]:=\{1, \ldots, m\}$ is the Poincaré dual of $\cap_{i \in I} M_{i}$, we see that

$$
\prod_{i \in I} \tau_{i}=0 \quad \text { if } \quad \cap_{i \in I} M_{i}=\emptyset .
$$

It turns out that $H_{T}^{*}(M)$ is generated by $\tau_{i}$ 's as a ring and that the relations in (3.2) are the only relations among $\tau_{i}$ 's, i.e., we have

Lemma 3.1. $H_{T}^{*}(M)=\mathbb{Z}\left[\tau_{1}, \ldots, \tau_{m}\right] /\left(\prod_{i \in I} \tau_{i} \mid \cap_{i \in I} M_{i}=\emptyset\right)$ as rings.

In particular, $\tau_{i}$ 's are a free additive basis of $H_{T}^{2}(M)$ and the following easily follows from this fact.

Lemma 3.2 (see [10, Lemma 1.5] for example). Let $\pi: E T \times_{T} M \rightarrow B T$ be the projection on the first factor. Then for each $i=1, \ldots, m$, there is a unique element $v_{i} \in H_{2}(B T)$ such that

$$
\pi^{*}(u)=\sum_{i=1}^{m}\left\langle u, v_{i}\right\rangle \tau_{i} \quad \text { for any } u \in H^{2}(B T),
$$

where $\langle$,$\rangle denotes the natural pairing between cohomology and homology.$

The Leray-Serre spectral sequence of the fibration

$$
M \stackrel{\iota}{\longrightarrow} E T \times_{T} M \stackrel{\pi}{\longrightarrow} B T
$$

collapses because $H^{\text {odd }}(M)=H^{\text {odd }}(B T)=0$. Therefore $H_{T}^{*}(M)=H^{*}$ $(B T) \otimes H^{*}(M)$ as $H^{*}(B T)$-modules and hence $H^{*}(M)$ is the quotient of $H_{T}^{*}(M)$ by the ideal generated by $\pi^{*}(u)$ for $u \in H^{2}(B T)$. This together with Lemmas 3.1 and 3.2 implies the following well-known fact.

Proposition 3.1. We set

$$
\mu_{i}:=\iota^{*}\left(\tau_{i}\right) \in H^{2}(M) .
$$

Then $H^{*}(M)$ is the quotient of a polynomial ring $\mathbb{Z}\left[\mu_{1}, \ldots, \mu_{m}\right]$ by the ideal generated by the following two types of elements:

(1) $\prod_{i \in I} \mu_{i}$ for $I \subset[m]$ with $\cap_{i \in I} M_{i}=\emptyset$. 
(2) $\sum_{i=1}^{m}\left\langle u, v_{i}\right\rangle \mu_{i}$ for $u \in H^{2}(B T)$.

A homomorphism $f: S^{1} \rightarrow T$ induces a continuous map $B f: B S^{1} \rightarrow$ $B T$. We fix a generator $\kappa$ of $H_{2}\left(B S^{1}\right) \cong \mathbb{Z}$. Then the correspondence $f \rightarrow$ $(B f)_{*}(\kappa)$ defines an isomorphism

$$
\operatorname{Hom}\left(S^{1}, T\right) \cong H_{2}(B T)
$$

and we denote by $\lambda_{v}$ the element of $\operatorname{Hom}\left(S^{1}, T\right)$ corresponding to $v \in$ $H_{2}(B T)$. The identity in Lemma 3.2 implies the following.

Lemma 3.3 (see [10, Lemma 1.10] for example). For the elements $v_{i} \in$ $H_{2}(B T)(i=1, \ldots, m)$ defined in Lemma $3.2, \lambda_{v_{i}}\left(S^{1}\right)$ is the circle subgroup of $T$ which fixes $M_{i}$ pointwise.

This lemma corresponds to Lemma 2.1. More precisely, one can see that the $v_{i}$ 's defined in Lemma 3.2 can be identified with the $v_{i}$ 's in Section 2 through an identification

$$
H_{2}(B T)=\mathbb{Z}^{n},
$$

(see [10] for example). Taking the dual of this identification, we obtain an identification

$$
H^{2}(B T)=\left(\mathbb{Z}^{n}\right)^{*}
$$

Then (2.1) can be rewritten as

$$
P=\left\{u \in H^{2}(B T ; \mathbb{R}) \mid\left\langle u, v_{i}\right\rangle \geq a_{i} \quad(i=1, \ldots, m)\right\},
$$

where $\langle$,$\rangle denotes the natural pairing between cohomology and homology$ as before.

Lemma 3.4. The exact sequences in (2.2) and (2.3) can be regarded as exact sequences derived from the fibration (3.4), namely, (2.2) can be regarded as

$$
0 \rightarrow H_{2}(M) \stackrel{\iota_{*}}{\longrightarrow} H_{2}^{T}(M) \stackrel{\pi_{*}}{\longrightarrow} H_{2}(B T) \rightarrow 0
$$

and (2.3) as

$$
0 \leftarrow H^{2}(M) \stackrel{\iota^{*}}{\longleftarrow} H_{T}^{2}(M) \stackrel{\pi^{*}}{\longleftarrow} H^{2}(B T) \leftarrow 0 .
$$

Proof. If we identify $H_{T}^{2}(M)$ with $\left(\mathbb{Z}^{m}\right)^{*}$ through the identification of $\tau_{i}$ with $e_{i}^{*}$ for $i=1, \ldots, m$, then (3.3) agrees with (2.4) and this implies the lemma.

Through the identifications in Lemma 3.4, (2.7) turns into

$$
\mathcal{Z}_{P}=\left\{z \in \mathbb{C}^{m} \mid \sum_{i=1}^{m}\left(\frac{1}{2}\left|z_{i}\right|^{2}+a_{i}\right) \mu_{i}=0\right\},
$$

where $\mu_{i}$ 's are the elements of $H^{2}(M)$ defined in (3.5). 


\section{Roots of a compact Lie subgroup of $\operatorname{Symp}(M, \omega)$}

If $g \in \operatorname{Symp}(M, \omega)$ normalizes the torus $T$, then $\rho_{g}$ defined by

$$
\rho_{g}(t):=g t g^{-1}
$$

is a group automorphism of $T$ and the diffeomorphism $g$ of $M$ is $\rho_{g}$-equivariant. Let $E \rho_{g}$ be a homeomorphism of $E T$ induced from $\rho_{g}$. It is $\rho_{g}$-equivariant, i.e., $E \rho_{g}(e t)=E \rho_{g}(e) \rho_{g}(t)$ for $e \in E T$ and $t \in T$. Therefore, the homeomorphism of $E T \times M$ sending $(e, x)$ to $\left(E \rho_{g}(e), g x\right)$ is $\rho_{g}$-equivariant and induces a homeomorphism of $E T \times_{T} M$. Hence we obtain a ring automorphism of $H_{T}^{*}(M)$, denoted by $g^{*}$, which preserves the subalgebra $\pi^{*}\left(H^{*}(B T)\right)$. It easily follows from the definition of $g^{*}$ that

$$
g^{*} \circ \pi^{*}=\pi^{*} \circ \rho_{g}^{*} \quad \text { on } H^{*}(B T),
$$

where $\rho_{g}{ }^{*}$ is an automorphism of $H^{*}(B T)$ induced from $\rho_{g}$.

Since the diffeomorphism $g$ of $M$ is $\rho_{g}$-equivariant, it permutes the characteristic submanifolds $M_{i}$ 's. Moreover, since $g$ preserves the form $\omega$, it preserves the orientations on $M$ and $M_{i}$ 's induced from $\omega$. These imply that there is a permutation $\sigma$ on $[m]$ such that

$$
g^{*}\left(\tau_{i}\right)=\tau_{\sigma(i)} \text { for any } i .
$$

With these understood

Lemma 4.1. Let $\rho_{g_{*}}$ be an automorphism of $H_{*}(B T)$ induced from $\rho_{g}$. Then $\rho_{g_{*}}\left(v_{\sigma(i)}\right)=v_{i}$ for any $i$.

Proof. Applying $g^{*}$ to the left- and right-hand sides of the identity (3.3), it follows from (4.2) and (4.3) that we have

$$
\pi^{*}\left(\rho_{g}{ }^{*}(u)\right)=g^{*}\left(\pi^{*}(u)\right)=\sum_{i=1}^{m}\left\langle u, v_{i}\right\rangle g^{*}\left(\tau_{i}\right)=\sum_{i=1}^{m}\left\langle u, v_{i}\right\rangle \tau_{\sigma(i)},
$$

while it follows from (3.3) applied to $\rho_{g}{ }^{*}(u)$ instead of $u$ that we have

$$
\begin{aligned}
\pi^{*}\left(\rho_{g}{ }^{*}(u)\right) & =\sum_{i=1}^{m}\left\langle\rho_{g}{ }^{*}(u), v_{i}\right\rangle \tau_{i}=\sum_{i=1}^{m}\left\langle\rho_{g}{ }^{*}(u), v_{\sigma(i)}\right\rangle \tau_{\sigma(i)} \\
& =\sum_{i=1}^{m}\left\langle u, \rho_{g_{*}}\left(v_{\sigma(i)}\right)\right\rangle \tau_{\sigma(i)} .
\end{aligned}
$$

Comparing (4.4) with (4.5) and noting that $\tau_{\sigma(i)}$ 's are free over $\mathbb{Z}$, we obtain

$$
\left\langle u, v_{i}\right\rangle=\left\langle u, \rho_{g_{*}}\left(v_{\sigma(i)}\right)\right\rangle \quad \text { for any } i,
$$

but since this identity holds for any $u \in H^{2}(B T)$, the desired identity in the lemma follows. 
The following lemma is due to $M$. Wiemeler and will play a key role in the subsequent argument.

Lemma 4.2. [12, Lemma 2.1]. If $g$ induces the identity on $H^{2}(M)$ and $\rho_{g}{ }^{*}$ is a reflection on $H^{2}(B T)$, then the $\sigma$ in (4.3) permutes exactly two elements in $[m]$ and fixes the others.

Proof. Since $\rho_{g}{ }^{*}$ is a reflection, its trace is $n-2$. On the other hand, since $g$ induces the identity on $H^{2}(M)$ by assumption, the trace of $g^{*}$ on $H_{T}^{2}(M)$ must be $m-2$ by (3.9). However, $H_{T}^{2}(M)$ is freely generated by $\tau_{i}$ 's over $\mathbb{Z}$ and $g^{*}$ permutes the generators by (4.3), so the lemma follows.

Dualizing the isomorphism (3.6), we obtain an isomorphism

$$
\operatorname{Hom}\left(T, S^{1}\right) \cong H^{2}(B T)
$$

For $u \in H^{2}(B T)$, we denote by $\chi^{u} \in \operatorname{Hom}\left(T, S^{1}\right)$ the element corresponding to $u$ through the isomorphism (4.6).

Now we take a compact Lie subgroup $G$ of $\operatorname{Symp}(M, \omega)$ containing $T$ and denote by $G^{0}$ the identity component of $G$. As remarked in the Introduction, the torus $T$ is a maximal torus of $G$.

Definition 4.1. A root of $G$ is a non-zero weight of the adjoint representation of $T$ on $\mathfrak{g} \otimes \mathbb{C}$, where $\mathfrak{g}$ denotes the Lie algebra of $G$. We think of a root of $G$ as an element of $H^{2}(B T)$ through the isomorphism (4.6) and denote by $\Delta(G)$ the root system of $G$, that is the set of roots of $G$. Needless to say, $\Delta(G)$ depends only on the identity component $G^{0}$.

For $\alpha \in \Delta(G)$, we denote by $T_{\alpha}$ the identity component of the kernel of $\chi^{\alpha}: T \rightarrow S^{1}$. Since $\alpha$ is non-zero, $T_{\alpha}$ is a codimension 1 subtorus of $T$. Let $G_{\alpha}^{0}$ be the identity component of the subgroup of $G^{0}$ which commutes with $T_{\alpha}$. The group $N_{G_{\alpha}^{0}}(T) / T$ is of order two and let $g \in N_{G_{\alpha}^{0}}(T)$ be a representative of the non-trivial element in $N_{G_{\alpha}^{0}}(T) / T$. The automorphism $\rho_{g}$ of $T$ is independent of the choice of the representative $g$, so we may denote it by $\rho_{\alpha}$. It is of order two, its fixed point set contains the codimension 1 subtorus $T_{\alpha}$ and $\rho_{\alpha}^{*}(\alpha)=-\alpha$. We note that $\rho_{\alpha}^{*}$ is the Weyl group action associated with $\alpha \in \Delta(G)$.

Similarly, $\rho_{\alpha_{*}}$ is a reflection on $H_{2}(B T)$ and we note that

$$
\operatorname{Fix}\left(\rho_{\alpha_{*}}\right)=H_{2}\left(B T_{\alpha}\right)=\operatorname{Ker} \alpha
$$

Lemma 4.3. For $\alpha \in \Delta(G)$, there are $i, j \in[m]$ such that

$$
\left\langle\alpha, v_{i}\right\rangle=-\left\langle\alpha, v_{j}\right\rangle(\neq 0) \text { and }\left\langle\alpha, v_{k}\right\rangle=0 \quad \text { for any } k \neq i, j \text {. }
$$


Proof. Let $g \in N_{G_{\alpha}^{0}}(T)$ be a representative of the non-trivial element in $N_{G_{\alpha}^{0}}(T) / T$. Since $g$ is in $G^{0}$, it is homotopic to the identity so that $g$ induces the identity on $H^{2}(M)$. Moreover, $\rho_{g}^{*}=\rho_{\alpha}^{*}$ is a reflection as observed above. Therefore there are $i, j \in[m]$ such that

$$
g^{*}\left(\tau_{i}\right)=\tau_{j}, \quad g^{*}\left(\tau_{j}\right)=\tau_{i}, \quad g^{*}\left(\tau_{k}\right)=\tau_{k} \quad \text { for any } k \neq i, j
$$

by Lemma 4.2. It follows from Lemma 4.1 that

$$
\rho_{\alpha *}\left(v_{i}\right)=v_{j}, \quad \rho_{\alpha *}\left(v_{j}\right)=v_{i}, \quad \rho_{\alpha *}\left(v_{k}\right)=v_{k} \quad \text { for any } k \neq i, j
$$

and hence $\left\langle\alpha, v_{k}\right\rangle=0$ for $k \neq i, j$ by (4.7). Finally, since $\rho_{\alpha}^{*}(\alpha)=-\alpha$, we have

$$
\left\langle\alpha, v_{i}\right\rangle=-\left\langle\rho_{\alpha}^{*}(\alpha), v_{i}\right\rangle=-\left\langle\alpha, \rho_{\alpha *}\left(v_{i}\right)\right\rangle=-\left\langle\alpha, v_{j}\right\rangle,
$$

proving the lemma.

Lemma 4.4. For $\alpha, \beta \in \Delta(G)$ we have

$$
\rho_{\alpha}^{*}(\beta)=\beta-\frac{\left\langle\beta, v_{i}\right\rangle-\left\langle\beta, v_{j}\right\rangle}{\left\langle\alpha, v_{i}\right\rangle} \alpha=\beta-\frac{\left\langle\beta, v_{j}\right\rangle-\left\langle\beta, v_{i}\right\rangle}{\left\langle\alpha, v_{j}\right\rangle} \alpha .
$$

Proof. It follows from (4.9) that

$$
\begin{aligned}
& \left\langle\rho_{\alpha}^{*}(\beta)-\beta, v_{i}\right\rangle=\left\langle\beta, \rho_{\alpha *}\left(v_{i}\right)\right\rangle-\left\langle\beta, v_{i}\right\rangle=\left\langle\beta, v_{j}\right\rangle-\left\langle\beta, v_{i}\right\rangle, \\
& \left\langle\rho_{\alpha}^{*}(\beta)-\beta, v_{j}\right\rangle=\left\langle\beta, \rho_{\alpha *}\left(v_{j}\right)\right\rangle-\left\langle\beta, v_{j}\right\rangle=\left\langle\beta, v_{i}\right\rangle-\left\langle\beta, v_{j}\right\rangle, \\
& \left\langle\rho_{\alpha}^{*}(\beta)-\beta, v_{k}\right\rangle=\left\langle\beta, \rho_{\alpha *}\left(v_{k}\right)\right\rangle-\left\langle\beta, v_{k}\right\rangle=0 \quad \text { for } k \neq i, j .
\end{aligned}
$$

This together with (4.8) shows that the three terms in the lemma take a same value on each $v_{\ell}$. Since $v_{\ell}$ 's span $H_{2}(B T)$, the desired identity in the lemma follows.

Let $a_{\beta, \alpha}$ be the constant defined by

$$
\rho_{\alpha}^{*}(\beta)=\beta-a_{\beta, \alpha} \alpha .
$$

By Lemma 4.4 we have

$$
a_{\beta, \alpha}=\frac{\left\langle\beta, v_{i}\right\rangle-\left\langle\beta, v_{j}\right\rangle}{\left\langle\alpha, v_{i}\right\rangle}=\frac{\left\langle\beta, v_{j}\right\rangle-\left\langle\beta, v_{i}\right\rangle}{\left\langle\alpha, v_{j}\right\rangle} .
$$

The following is well known (see $[\mathbf{9}, 9.4]$ but $a_{\beta, \alpha}$ is denoted $\langle\beta, \alpha\rangle$ in the book):

(1) $a_{\beta, \alpha}$ is an integer,

(2) $a_{\beta, \alpha} \neq 0$ if and only if $a_{\alpha, \beta} \neq 0$,

(3) $0 \leq a_{\beta, \alpha} a_{\alpha, \beta} \leq 3$ if $\beta \neq \pm \alpha$.

We set

$$
N_{\alpha}:=\left|\left\langle\alpha, v_{i}\right\rangle\right|=\left|\left\langle\alpha, v_{j}\right\rangle\right| .
$$


We say that $\alpha$ and $\beta$ are joined if $\rho_{\alpha}^{*}(\beta) \neq \beta$ (i.e., $a_{\beta, \alpha} \neq 0$ ). By (2) above, $\rho_{\alpha}^{*}(\beta) \neq \beta$ if and only if $\rho_{\beta}^{*}(\alpha) \neq \alpha$.

Lemma 4.5. If $\alpha$ and $\beta$ are joined, then $N_{\alpha}=N_{\beta}$.

Proof. Since $N_{-\alpha}=N_{\alpha}$, we may assume $\beta \neq \pm \alpha$. Then $a_{\beta, \alpha}$ is a non-zero integer, so it follows from (4.11) that $\left|a_{\beta, \alpha}\right| \geq 2$ if $N_{\alpha} \neq N_{\beta}$ (note that since $\beta \neq \pm \alpha$, either $\left\langle\beta, v_{i}\right\rangle$ or $\left\langle\beta, v_{j}\right\rangle$ is 0 by Lemma 4.3). Changing the role of $\alpha$ and $\beta$, we also have that $\left|a_{\alpha, \beta}\right| \geq 2$ if $N_{\alpha} \neq N_{\beta}$. But this contradicts the above fact (3) that $0 \leq a_{\beta, \alpha} a_{\alpha, \beta} \leq 3$ if $\beta \neq \pm \alpha$.

$\Delta(G)$ decomposes into a direct sum of irreducible root systems. Since we are concerned with the isomorphism type of $\Delta(G)$ as a root system, we may assume that $N_{\alpha}=1$ for any $\alpha$ by Lemma 4.5.

\section{The root system of a moment polytope}

Remember that the correspondence from symplectic toric manifolds to their moment polytopes (which are non-singular) is one-to-one. Motivated by the observation made in Section 4, we make the following definition.

Definition 5.1. For a non-singular polytope $P$ described in (3.7), we define

$$
\begin{aligned}
R(P):= & \left\{\alpha \in H^{2}(B T) \mid \quad\left\langle\alpha, v_{i}\right\rangle=1,\left\langle\alpha, v_{j}\right\rangle=-1 \text { for some } i, j,\right. \\
& \text { and } \left.\left\langle\alpha, v_{k}\right\rangle=0 \quad \text { for } k \neq i, j\right\},
\end{aligned}
$$

and call it the root system of $P$. (It will be proved below that $R(P)$ is actually a root system.)

Remark 5.1. The root system $R(P)$ depends only on the $v_{i}$ 's and not on the constants $a_{i}$ 's used to describe the moment polytope $P$ in (3.7).

Example. We identify $H_{2}(B T)$ with $\mathbb{Z}^{n}$ and denote by $\left\{e_{i}\right\}_{i=1}^{n}$ the standard basis of $\mathbb{Z}^{n}$ and by $\left\{e_{i}^{*}\right\}_{i=1}^{n}$ the basis of $\left(\mathbb{Z}^{n}\right)^{*}$ dual to $\left\{e_{i}\right\}_{i=1}^{n}$. Remember that $m$ is the number of facets of $P$.

(1) Let $m=n+1$ and take $v_{i}=e_{i}$ for $1 \leq i \leq n$ and $v_{n+1}=-\sum_{i=1}^{n} e_{i}$. Then

$$
R(P)=\left\{ \pm e_{i}^{*}(1 \leq i \leq n), \quad \pm\left(e_{i}^{*}-e_{j}^{*}\right)(1 \leq i<j \leq n)\right\}
$$

and this is a root system of type $A_{n}$. Note that the manifold $M$ is the complex projective space $\mathbb{C} P^{n}$ of complex dimension $n$ in this case.

(2) Let $n=2, m=4$ and take $v_{1}=e_{1}, v_{2}=e_{2}, v_{3}=-e_{1}+a e_{2}$ and $v_{4}=-e_{2}$ where $a$ is an arbitrary integer. If $a=0$, then $P$ is a rectangle and

$$
R(P)=\left\{ \pm e_{1}^{*}, \pm e_{2}^{*}\right\},
$$

which is of type $A_{1} \times A_{1}$, and if $a \neq 0$, then $P$ is a trapezoid with two right angle corners and

$$
R(P)=\left\{ \pm e_{1}^{*}\right\}
$$


which is of type $A_{1}$. Note that the manifold $M$ is a Hirzebruch surface in this case.

(3) If $n=2$ and $m \geq 5$, then one easily checks that $R(P)$ is empty.

For $\alpha \in R(P)$ with $\left\langle\alpha, v_{i}\right\rangle=1$ and $\left\langle\alpha, v_{j}\right\rangle=-1$, we define a reflection $r_{\alpha}$ on $H_{2}(B T)$ by

$$
r_{\alpha}(v):=v-\langle\alpha, v\rangle\left(v_{i}-v_{j}\right),
$$

which interchanges $v_{i}$ and $v_{j}$ and fixes $v_{k}$ 's for $k \neq i, j$, and define its dual reflection $r_{\alpha}^{\vee}$ on $H^{2}(B T)$ by

$$
\left\langle r_{\alpha}^{\vee}(\beta), v\right\rangle:=\left\langle\beta, r_{\alpha}(v)\right\rangle=\left\langle\beta, v-\langle\alpha, v\rangle\left(v_{i}-v_{j}\right)\right\rangle .
$$

This shows that

$$
r_{\alpha}^{\vee}(\beta)=\beta-\left(\left\langle\beta, v_{i}\right\rangle-\left\langle\beta, v_{j}\right\rangle\right) \alpha .
$$

In particular, $r_{\alpha}^{\vee}( \pm \alpha)=\mp \alpha$. One can easily check that $r_{\alpha}^{\vee}$ preserves $R(P)$. Comparing Lemma 4.4 with (5.1) and noting that we may assume $N_{\alpha}=1$ for any $\alpha$, we see that $\rho_{\alpha}{ }^{*}$ agrees with $r_{\alpha}^{\vee}$ and hence we obtain the following.

Proposition 5.1. $R(P)$ is a root system and if $P$ is the moment polytope associated with a symplectic toric manifold $(M, \omega)$ and $G$ is a compact Lie subgroup of $\operatorname{Symp}(M, \omega)$ containing the torus $T$, then the root system $\Delta(G)$ of $G$ is a subsystem of $R(P)$.

We define a symmetric scalar product $\left(\right.$, ) on $H^{2}(B T)$ by

$$
(\beta, \gamma):=\sum_{\ell=1}^{m}\left\langle\beta, v_{\ell}\right\rangle\left\langle\gamma, v_{\ell}\right\rangle
$$

One easily sees from the definition of $R(P)$ that

$$
\begin{aligned}
& (\alpha, \alpha)=2 \text { for } \alpha \in R(P), \\
& (\alpha, \beta)=0 \text { or } \pm 1 \quad \text { for } \alpha, \beta \in R(P) \text { with } \beta \neq \pm \alpha .
\end{aligned}
$$

The group generated by the reflections $r_{\alpha}^{\vee}(\alpha \in R(P))$ is called the Weyl group of $R(P)$.

Lemma 5.1. The scalar product (, ) is invariant under the Weyl group of $R(P)$.

Proof. It suffices to check that $\left(r_{\alpha}^{\vee}(\beta), r_{\alpha}^{\vee}(\gamma)\right)=(\beta, \gamma)$ for $\alpha \in R(P)$ and $\beta, \gamma \in H^{2}(B T)$. By definition there are $i, j \in[m]$ such that $\left\langle\alpha, v_{i}\right\rangle=1$, $\left\langle\alpha, v_{j}\right\rangle=-1$ and $\left\langle\alpha, v_{k}\right\rangle=0$ for $k \neq i, j$. We denote $\left\langle\eta, v_{\ell}\right\rangle$ by $\eta_{\ell}$ for 
$\eta \in H^{2}(B T)$. Then since $(\alpha, \eta)=\eta_{i}-\eta_{j}$, it follows from (5.1) that

$$
\begin{aligned}
\left(r_{\alpha}^{\vee}(\beta), r_{\alpha}^{\vee}(\gamma)\right)= & \left(\beta-\left(\beta_{i}-\beta_{j}\right) \alpha, \gamma-\left(\gamma_{i}-\gamma_{j}\right) \alpha\right) \\
= & (\beta, \gamma)-\left(\beta_{i}-\beta_{j}\right)(\alpha, \gamma)-\left(\gamma_{i}-\gamma_{j}\right)(\beta, \alpha) \\
& +\left(\beta_{i}-\beta_{j}\right)\left(\gamma_{i}-\gamma_{j}\right)(\alpha, \alpha) \\
= & (\beta, \gamma)-\left(\beta_{i}-\beta_{j}\right)\left(\gamma_{i}-\gamma_{j}\right)-\left(\gamma_{i}-\gamma_{j}\right)\left(\beta_{i}-\beta_{j}\right) \\
& +2\left(\beta_{i}-\beta_{j}\right)\left(\gamma_{i}-\gamma_{j}\right) \\
= & (\beta, \gamma),
\end{aligned}
$$

proving the lemma.

For $\alpha, \beta \in R(P)$ we define an integer $a_{\beta, \alpha}$ by

$$
r_{\alpha}^{\vee}(\beta)=\beta-a_{\beta, \alpha} \alpha
$$

similarly to (4.10). If $\left\langle\alpha, v_{i}\right\rangle=1$ and $\left\langle\alpha, v_{j}\right\rangle=-1$, then

$$
a_{\beta, \alpha}=\left\langle\beta, v_{i}\right\rangle-\left\langle\beta, v_{j}\right\rangle
$$

by (5.1). Another description of $a_{\beta, \alpha}$ is the following.

Lemma 5.2. $a_{\beta, \alpha}=(\alpha, \beta)$ for $\alpha, \beta \in R(P)$. In particular, $a_{\beta, \alpha}=a_{\alpha, \beta}$.

Proof. Since $r_{\alpha}^{\vee}(\alpha)=-\alpha$ and $r_{\alpha}^{\vee}$ is of order 2, it follows from Lemma 5.1 that

$$
\left(\alpha, r_{\alpha}^{\vee}(\beta)+\beta\right)=\left(r_{\alpha}^{\vee}(\alpha), r_{\alpha}^{\vee}\left(r_{\alpha}^{\vee}(\beta)+\beta\right)\right)=\left(-\alpha, \beta+r_{\alpha}^{\vee}(\beta)\right)
$$

and hence $\left(\alpha, r_{\alpha}^{\vee}(\beta)+\beta\right)=0$. This together with (5.3) and (5.4) implies the lemma.

Theorem 5.1. Any irreducible subsystem of $R(P)$ is of type $A$.

Proof. Let $\Phi$ be an irreducible subsystem of $R(P)$. The Cartan matrix $C(\Phi)$ of $\Phi$ is $\left(a_{\beta, \alpha}\right)$ where $\alpha$ and $\beta$ run over elements in a basis of $\Phi$. The diagonal entiries of $C(\Phi)$ are all 2 by (5.3) and $C(\Phi)$ is symmetric by Lemma 5.2. Therefore, $\Phi$ must be either of type $A, D$ or $E$ (see [9, p. 59]).

Suppose that $\Phi$ is of type $D$ or $E$. Then there are elements $\alpha, \beta, \gamma, \delta$ in the basis of $\Phi$ such that

$$
a_{\beta, \alpha}=a_{\gamma, \alpha}=a_{\delta, \alpha}=-1 .
$$

As before, let $v_{i}, v_{j}$ be the elements such that $\left\langle\alpha, v_{i}\right\rangle=1$ and $\left\langle\alpha, v_{j}\right\rangle=-1$. It follows from (5.5) and (5.6) that the values which $\beta, \gamma, \delta$ take on $v_{i}$ and $v_{j}$ must be either $(-1,0)$ or $(0,1)$. Therefore two of $\beta, \gamma, \delta$, say $\beta$ and $\gamma$, must take the same values on $v_{i}$ and $v_{j}$, say $(0,1)$. (The same argument below will work for $(-1,0)$.) Let $v_{k}$ be the other element on which $\beta$ takes a nonzero value. Then $r_{\beta}\left(v_{j}\right)=v_{k}$ and since $\left\langle\beta, v_{j}\right\rangle=1$, we have $\left\langle\beta, v_{k}\right\rangle=-1$. 
Moreover, since $\left\langle\gamma, v_{j}\right\rangle=1$ and $\beta \neq \gamma$, we have $\left\langle\gamma, v_{k}\right\rangle=0$. Therefore,

$$
\begin{aligned}
& \left\langle r_{\beta}^{\vee}(\gamma)-\gamma, v_{j}\right\rangle=\left\langle\gamma, r_{\beta}\left(v_{j}\right)\right\rangle-\left\langle\gamma, v_{j}\right\rangle=\left\langle\gamma, v_{k}\right\rangle-\left\langle\gamma, v_{j}\right\rangle=-1, \\
& \left\langle r_{\beta}^{\vee}(\gamma)-\gamma, v_{k}\right\rangle=\left\langle\gamma, r_{\beta}\left(v_{k}\right)\right\rangle-\left\langle\gamma, v_{k}\right\rangle=\left\langle\gamma, v_{j}\right\rangle-\left\langle\gamma, v_{k}\right\rangle=1 .
\end{aligned}
$$

Since $\beta$ takes 1 on $v_{j}$ and -1 on $v_{k}$, the above shows that $r_{\beta}^{\vee}(\gamma)-\gamma=-\beta$ and hence $a_{\gamma, \beta}=1$ by (5.4). However $a_{\gamma, \beta}$ must be non-positive because $\beta$ and $\gamma$ are in the basis of $\Phi$ and $\beta \neq \gamma$. This is a contradiction. Thus $\Phi$ is neither of type $D$ nor $E$ and hence of type $A$.

We conclude this section with the following corollary which follows from Proposition 5.1 and Theorem 5.1.

Corollary 5.1. If $G$ is a compact Lie subgroup of $\operatorname{Symp}(M, \omega)$ containing the torus $T$, then any irreducible factor of $\Delta(G)$ is of type $A$.

\section{Connected maximal compact Lie subgroup of $\operatorname{Symp}(M, \omega)$}

In this section we shall observe that the equality $\Delta(G)=R(P)$ is attained for some compact connected Lie subgroup $G$ of $\operatorname{Symp}(M, \omega)$.

As discussed in Section 2, we may think of $M$ as $\mathcal{Z}_{P} / \operatorname{Ker} \mathcal{V}$ and $\omega$ as the form induced from the standard form $\omega_{0}$ on $\mathbb{C}^{m}$, where

$$
\mathcal{Z}_{P}=\left\{z \in \mathbb{C}^{m} \mid \sum_{i=1}^{m}\left(\frac{1}{2}\left|z_{i}\right|^{2}+a_{i}\right) \mu_{i}=0\right\}
$$

from (3.10) and

$$
\operatorname{Ker} \mathcal{V}=\left\{\left(g_{1}, \ldots, g_{m}\right) \in\left(S^{1}\right)^{m} \mid \prod_{i=1}^{m} g_{i}^{\left\langle u, v_{i}\right\rangle}=1 \text { for } \forall u \in H^{2}(B T)\right\}
$$

from (2.13) through the identification $H^{2}(B T)=\mathbb{Z}^{n}$ discussed in Section 3 .

Lemma 6.1. Let $\alpha$ be an element of $R(P)$ such that $\left\langle\alpha, v_{i}\right\rangle=1,\left\langle\alpha, v_{j}\right\rangle=-1$ and $\left\langle\alpha, v_{k}\right\rangle=0$ for $k \neq i, j$. Then $\mu_{i}=\mu_{j}$ in (6.1) and $g_{i}=g_{j}$ in (6.2).

Proof. It follows from (3.3) that $\pi^{*}(\alpha)=\tau_{i}-\tau_{j}$. Applying $\iota^{*}$ to the both sides of this identity, we get the former identity in the lemma because $\iota^{*} \circ \pi^{*}=0$ by (3.9) and $\mu_{\ell}=\iota^{*}\left(\tau_{\ell}\right)$ by (3.5). If we take the $\alpha$ as $u$ in (6.2), then the condition $\prod_{i=1}^{m} g_{i}^{\left\langle u, v_{i}\right\rangle}=1$ reduces to $g_{i} g_{j}^{-1}=1$ and this proves the latter statement in the lemma.

The purpose of this section is to prove the following.

Proposition 6.1. There is a closed connected subgroup $\tilde{G}$ of the unitary group $U(m)$, which leaves $\mathcal{Z}_{P}$ invariant and contains $\operatorname{Ker} \mathcal{V}$ in its center (so that the action of $\tilde{G}$ induces an effective action of $\tilde{G} / \operatorname{Ker} \mathcal{V}$ on $M=$ $\left.\mathcal{Z}_{P} / \operatorname{Ker} \mathcal{V}\right)$ and $\Delta(\tilde{G} / \operatorname{Ker} \mathcal{V})=R(P)$. 
Proof. Let $\Phi$ be an irreducible factor of $R(P)$. It is of type $A$ by Theorem 5.1. Suppose that the rank of $\Phi$ is $r-1$. Then it follows from Lemma 6.1 that there is a subset $I(\Phi):=\left\{i_{1}, \ldots, i_{r}\right\}$ of $[m]$ such that $\mu_{i_{1}}=\cdots=\mu_{i_{r}}$ and $g_{i_{1}}=\cdots=g_{i_{r}}$ for $g=\left(g_{1}, \ldots, g_{m}\right) \in \operatorname{Ker} \mathcal{V}$. Therefore the action of $U(m)$ on $\mathbb{C}^{m}$ restricted to the subgroup

$$
U(\Phi):=\left\{\left(x_{i j}\right) \in U(m) \mid x_{i j}=\delta_{i j} \text { unless both } i \text { and } j \text { are in } I(\Phi)\right\},
$$

where $\delta_{i j}=1$ if $i=j$ and 0 otherwise, leaves $\mathcal{Z}_{P}$ invariant and $U(\Phi)$ commutes with $\operatorname{Ker} \mathcal{V}$. We note that the root system of $U(\Phi)$ is (isomorphic to) $\Phi$.

Now we decompose $R(P)$ into sum of irreducible factors $\Phi_{1}, \ldots, \Phi_{s}$. Then the subsets $I\left(\Phi_{1}\right), \ldots, I\left(\Phi_{s}\right)$ of $[m]$ are disjoint. We consider the subgroup $\tilde{G}$ of $U(m)$ generated by $\prod_{i=1}^{s} U\left(\Phi_{i}\right)$ and (the diagonal subgroup) $\left(S^{1}\right)^{m}$. Since $\left(S^{1}\right)^{m}$ contains $\operatorname{Ker} \mathcal{V}$, so does $\tilde{G}$. It follows from the observation above that $\tilde{G}$ commutes with Ker $\mathcal{V}$ and the action of $U(m)$ on $\mathbb{C}^{m}$ restricted to $\tilde{G}$ leaves $\mathcal{Z}_{P}$ invariant so that the action descends to an effective action of $\tilde{G} / \operatorname{Ker} \mathcal{V}$ on $M$. Since the action of $\tilde{G}$ on $\mathcal{Z}_{P}$ preserves the standard form $\omega_{0} \mid \mathcal{Z}_{P}$, the induced action of $\tilde{G} / \operatorname{Ker} \mathcal{V}$ on $M$ preserves the form $\omega$ on $M$, see (2.14). Finally, $\Delta(\tilde{G} / \operatorname{Ker} \mathcal{V})=R(P)$ by construction.

\section{Automorphisms of a moment polytope}

Since the dual of the Lie algebra of $T$ can be naturally identified with $H^{2}(B T ; \mathbb{R})$, we think of the moment map $\mu$ associated with $(M, \omega)$ as taking values in $H^{2}(B T ; \mathbb{R})$ and $P=\mu(M)$. When $g \in \operatorname{Symp}(M, \omega)$ normalizes the torus $T$, we associated the $\rho_{g}$ of $\operatorname{Aut}(T)$ to $g$ in Section 4 , where

$$
\rho_{g}(t)=g t g^{-1} \quad \text { for } t \in T
$$

and $\operatorname{Aut}(T)$ denotes the group of automorphisms of $T$. The moment map associated to $(M, \omega)$ with the $T$-action twisted by $\rho_{g}$ is given by $\rho_{g}{ }^{*} \circ \mu$, so the image of $M$ by the map is $\rho_{g}{ }^{*}(P)$. Since $g$ preserves the form $\omega$, the images of $M$ by $\mu$ and $\rho_{g}{ }^{*} \circ \mu$ are congruent modulo parallel translations in $H^{2}(B T ; \mathbb{R})$. Motivated by this observation, we define

$$
\operatorname{Aut}(P):=\left\{\rho \in \operatorname{Aut}(T) \mid \rho^{*}(P) \equiv P\right\},
$$

where $\equiv$ denotes congruence modulo parallel translations in $H^{2}(B T ; \mathbb{R})$.

Remark 7.1. As remarked before, the root system $R(P)$ depends only on the $v_{i}$ 's and not on the constants $a_{i}$ 's used to define the moment polytope $P$ in (2.1) or (3.7). However, Aut $(P)$ actually depends on the $a_{i}$ 's. For instance, $\operatorname{Aut}(P)$ for a square $P$ is (a dihedral group) of order 8 while $\operatorname{Aut}(P)$ for a (non-square) rectangle $P$ is of order 4 . 
The correspondence $g \rightarrow \rho_{g}$ defines a homomorphism

$$
\mathcal{D}: N_{G}(T) \rightarrow \operatorname{Aut}(P),
$$

where $G$ is any subgroup of $\operatorname{Symp}(M, \omega)$ containing $T$ (e.g., $G$ may be the entire group $\operatorname{Symp}(M, \omega))$ and $N_{G}(T)$ denotes the normalizer of $T$ in $G$. If $g \in T$, then $\rho_{g}$ is the identity; so $T$ is in the kernel of $\mathcal{D}$.

Lemma 7.1. If $G$ is a compact Lie subgroup of $\operatorname{Symp}(M, \omega)$ containing the torus $T$, then the kernel of $\mathcal{D}$ is exactly $T$.

Proof. We note that $g \in N_{G}(T)$ permutes the characteristic submanifolds $M_{i}$ 's of $M$. Suppose that $g \in N_{G}(T)$ is in the kernel of $\mathcal{D}$. Then $g$ maps $M_{i}$ to itself for each $i$. Let $x$ be a $T$-fixed point in $M$. Then $x=\bigcap_{i \in I} M_{i}$ for some $I \in[m]$ with cardinality $n$, so that $x$ is fixed by $g$. We decompose the tangent space $\tau_{x} M$ of $M$ at $x$ into

$$
\tau_{x} M=\bigoplus_{i \in I} \tau_{x} M / \tau_{x} M_{i}
$$

The differential $d g: \tau_{x} M \rightarrow \tau_{x} M$ preserves each real 2-dimensional eigenspace $\tau_{x} M / \tau_{x} M_{i}$ since $g$ fixes $x$ and maps $M_{i}$ to itself for each $i$. The symplectic form $\omega$ determines an orientation on $\tau_{x} M / \tau_{x} M_{i}$ for each $i$ and $d g$ preserves the orientation on $\tau_{x} M / \tau_{x} M_{i}$ since $g$ preserves the form $\omega$.

Since $G$ is compact, there exists a $G$-invariant Riemannian metric on $M$ so that we may assume that $d g$ is an orthogonal transformation on $\tau_{x} M / \tau_{x} M_{i}$ but since $d g$ preserves the orientation on it, $d g$ on $\tau_{x} M / \tau_{x} M_{i}$ is a rotation and hence there exists $t \in T$ such that $d g=d t$, i.e., $d\left(g t^{-1}\right)$ is the identity on $\tau_{x} M$. On the other hand, since $g t^{-1}$ is contained in $G$ and $G$ is compact, the fixed point set of $g t^{-1}$ is a closed submanifold of $M$. The connected component of this submanifold containing $x$ is of codimension 0 because $d\left(g t^{-1}\right)$ is the identity on $\tau_{x} M$. Since $M$ is connected, the connected component must agree with $M$ and this means $g=t$, proving the lemma.

Corollary 7.1. Let $G$ be a compact Lie subgroup of $\operatorname{Symp}(M, \omega)$ containing the torus $T$ and let $G^{0}$ be the identity component of $G$. Then

$$
G / G^{0} \cong \mathcal{D}\left(N_{G}(T)\right) / \mathcal{D}\left(N_{G^{0}}(T)\right) \subset \operatorname{Aut}(P) / \mathcal{D}\left(N_{G^{0}}(T)\right),
$$

where $\mathcal{D}$ is the map in (7.2) and $N_{G^{0}}(T)$ is the normalizer of $T$ in $G^{0}$.

Proof. Since $T$ is a maximal torus of $G$ and maximal tori in $G$ are conjugate to each other because $G$ is compact, the inclusion $N_{G}(T) \rightarrow G$ induces an isomorphism

$$
N_{G}(T) / N_{G^{0}}(T) \cong G / G^{0} .
$$

This fact together with Lemma 7.1 implies the corollary. 
We shall construct a cross section of the homomorphism $\mathcal{D}$ in (7.2) when $G=\operatorname{Symp}(M, \omega)$. We recall the description (3.7) of $P$ :

$$
P=\left\{u \in H^{2}(B T ; \mathbb{R}) \mid\left\langle u, v_{i}\right\rangle \geq a_{i} \quad(i=1, \ldots, m)\right\} .
$$

Let $\rho \in \operatorname{Aut}(P)$. Since $\rho^{*}(P) \equiv P$, we have

$$
\rho^{*}(P)=P+u_{0} \quad \text { for some } u_{0} \in H^{2}(B T ; \mathbb{R}) .
$$

On the other hand, we have

$$
\rho^{*}(P)=\left\{\rho^{*}(u) \in H^{2}(B T ; \mathbb{R}) \mid\left\langle u, v_{i}\right\rangle \geq a_{i} \quad(i=1, \ldots, m)\right\}
$$

by definition and this can be rewritten as

$$
\rho^{*}(P)=\left\{u \in H^{2}(B T ; \mathbb{R}) \mid\left\langle u, \rho_{*}{ }^{-1}\left(v_{i}\right)\right\rangle \geq a_{i} \quad(i=1, \ldots, m)\right\} .
$$

Since $\rho^{*}(P) \equiv P$, it follows from (7.4) and (7.5) that there exists a permutation $\sigma$ on $[m]$ such that

$$
\rho_{*}{ }^{-1}\left(v_{i}\right)=v_{\sigma(i)} \quad \text { for any } i \in[m]
$$

so that

$$
\rho^{*}(P)=\left\{u \in H^{2}(B T ; \mathbb{R}) \mid\left\langle u, v_{\sigma(i)}\right\rangle \geq a_{i} \quad(i=1, \ldots, m)\right\} .
$$

Therefore,

$$
\begin{aligned}
\left\langle u, v_{\sigma(i)}\right\rangle \geq a_{\sigma(i)} & \Longleftrightarrow u \in P \\
& \Longleftrightarrow u+u_{0} \in \rho^{*}(P) \\
& \Longleftrightarrow\left\langle u+u_{0}, v_{\sigma(i)}\right\rangle \geq a_{i} \\
& \Longleftrightarrow\left\langle u, v_{\sigma(i)}\right\rangle \geq a_{i}-\left\langle u_{0}, v_{\sigma(i)}\right\rangle
\end{aligned}
$$

and this shows that

$$
a_{\sigma(i)}=a_{i}-\left\langle u_{0}, v_{\sigma(i)}\right\rangle \text { for any } i .
$$

We make one more observation on the permutation $\sigma$.

Lemma 7.2. There is a ring automorphism of $H^{*}(M)$ sending $\mu_{i}$ to $\mu_{\sigma(i)}$ for each $i$.

Proof. Since $\sigma$ is induced from the automorphism $\rho$ of $P$, we note that $\cap_{i \in I} P_{i}=\emptyset$ if and only if $\cap_{j \in \sigma(I)} P_{j}=\emptyset$ for $I \subset[m]$. Furthermore, we note that $\cap_{i \in I} P_{i}=\emptyset$ if and only if $\cap_{i \in I} M_{i}=\emptyset$. Therefore, Lemma 3.1 ensures that sending $\tau_{i}$ to $\tau_{\sigma(i)}$ for each $i$ induces a ring automorphism of $H_{T}^{*}(M)$, which we denote by $f$. 
Applying $f$ to the both sides of (3.3), we have

$$
f\left(\pi^{*}(u)\right)=\sum_{i=1}^{m}\left\langle u, v_{i}\right\rangle f\left(\tau_{i}\right)=\sum_{i=1}^{m}\left\langle u, v_{i}\right\rangle \tau_{\sigma(i)},
$$

while it follows from (3.3) applied to $\rho^{*}(u)$ instead of $u$ that we have

$$
\begin{aligned}
\pi^{*}\left(\rho^{*}(u)\right) & =\sum_{i=1}^{m}\left\langle\rho^{*}(u), v_{i}\right\rangle \tau_{i}=\sum_{i=1}^{m}\left\langle\rho^{*}(u), v_{\sigma(i)}\right\rangle \tau_{\sigma(i)} \\
& =\sum_{i=1}^{m}\left\langle u, \rho_{*}\left(v_{\sigma(i)}\right)\right\rangle \tau_{\sigma(i)}=\sum_{i=1}^{m}\left\langle u, v_{i}\right\rangle \tau_{\sigma(i)},
\end{aligned}
$$

where we used (7.6) at the last identity. Comparing (7.8) with (7.9), we obtain the identity $f\left(\pi^{*}(u)\right)=\pi^{*}\left(\rho^{*}(u)\right)$ for any $u \in H^{2}(B T)$ and this shows that the ring automorphism $f$ of $H_{T}^{*}(M)$ preserves the subalgebra $\pi^{*}\left(H^{*}(B T)\right)$. Therefore $f$ induces a ring automorphism $\bar{f}$ of $H^{*}(M)$ by Proposition 3.1. Since $f\left(\tau_{i}\right)=\tau_{\sigma(i)}$ and $\mu_{i}=\iota^{*}\left(\tau_{i}\right)$ by $(3.5)$, we have $\bar{f}\left(\mu_{i}\right)=$ $\mu_{\sigma(i)}$ which proves the lemma.

We now define the unitary transformation $F_{\rho}$ of $\mathbb{C}^{m}$ by

$$
F_{\rho}\left(z_{1}, \ldots, z_{m}\right):=\left(z_{\sigma(1)}, \ldots, z_{\sigma(m)}\right) .
$$

It preserves $\mathcal{Z}_{P}$ because

$$
\begin{aligned}
z \in \mathcal{Z}_{P} & \Longleftrightarrow \sum\left(\frac{1}{2}\left|z_{\sigma(i)}\right|^{2}+a_{\sigma(i)}\right) \mu_{\sigma(i)}=0 \quad(\text { by }(3.10)) \\
& \left.\Longleftrightarrow \sum\left(\frac{1}{2}\left|z_{\sigma(i)}\right|^{2}+a_{i}-\left\langle u_{0}, v_{\sigma(i)}\right\rangle\right) \mu_{\sigma(i)}=0 \quad \text { (by }(7.7)\right) \\
& \Longleftrightarrow \sum\left(\frac{1}{2}\left|z_{\sigma(i)}\right|^{2}+a_{i}\right) \mu_{\sigma(i)}=0 \quad(\text { by }(2) \text { in Proposition 3.1) } \\
& \Longleftrightarrow \sum\left(\frac{1}{2}\left|z_{\sigma(i)}\right|^{2}+a_{i}\right) \mu_{i}=0 \quad(\text { by Lemma } 7.2) \\
& \Longleftrightarrow F_{\rho}(z) \in \mathcal{Z}_{P} \quad(\text { by }(3.10) \text { and }(7.10)) .
\end{aligned}
$$

Let $\phi$ be the automorphism of $\left(S^{1}\right)^{m}$ defined by

$$
\phi\left(g_{1}, \ldots, g_{m}\right):=\left(g_{\sigma(1)}, \ldots, g_{\sigma(m)}\right) .
$$

Then the map $F_{\rho}$ is $\phi$-equivariant.

Lemma 7.3. Let $\mathcal{V}:\left(S^{1}\right)^{m} \rightarrow T$ be the homomorphism in (2.11). Then $\mathcal{V} \circ \phi=\rho \circ \mathcal{V}$. In particular $\phi$ preserves $\operatorname{Ker} \mathcal{V}$. 
Proof. Noting that $\rho\left(\lambda_{v}(g)\right)=\lambda_{\rho_{*}(v)}(g)$, we see from (7.11), (2.11) and (7.6) that

$$
\begin{aligned}
\mathcal{V}\left(\phi\left(g_{1}, \ldots, g_{m}\right)\right) & =\mathcal{V}\left(g_{\sigma(1)}, \ldots, g_{\sigma(m)}\right)=\prod_{i=1}^{m} \lambda_{v_{i}}\left(g_{\sigma(i)}\right) \\
& =\prod_{i=1}^{m} \lambda_{\rho_{*}\left(v_{\sigma(i)}\right)}\left(g_{\sigma(i)}\right)=\rho\left(\prod_{i=1}^{m} \lambda_{v_{\sigma(i)}}\left(g_{\sigma(i)}\right)\right) \\
& =\rho\left(\prod_{i=1}^{m} \lambda_{v_{i}}\left(g_{i}\right)\right)=\rho\left(\mathcal{V}\left(g_{1}, \ldots, g_{m}\right)\right) .
\end{aligned}
$$

This proves the lemma.

Since $M=\mathcal{Z}_{P} / \operatorname{Ker} \mathcal{V}$ and $F_{\rho}$ is $\phi$-equivariant, $F_{\rho}$ induces a diffeomorphism $\bar{F}_{\rho}$ of $M$ by Lemma 7.3. By definition $F_{\rho}$ is a unitary transformation on $\mathbb{C}^{m}$, so $\bar{F}_{\rho}$ preserves the symplectic form $\omega$ and hence $\bar{F}_{\rho} \in \operatorname{Symp}(M, \omega)$.

Finally, we need to prove the following.

Lemma 7.4. $\bar{F}_{\rho}$ normalizes $T$ and $\mathcal{D}\left(\bar{F}_{\rho}\right)=\rho$.

Proof. We view an element $g=\left(g_{1}, \ldots, g_{m}\right)$ of $\left(S^{1}\right)^{m}$ as a diffeomorphism of $\mathcal{Z}_{P} \subset \mathbb{C}^{m}$. Then $F_{\rho} \circ g \circ F_{\rho}^{-1}=\phi(g)$. This identity decends to an identity

$$
\bar{F}_{\rho} \circ \mathcal{V}(g) \circ \bar{F}_{\rho}^{-1}=\mathcal{V}(\phi(g)) \quad \text { in } \operatorname{Symp}(M, \omega) .
$$

Since $T=\mathcal{V}\left(\left(S^{1}\right)^{m}\right)$, the identity (7.12) shows that $\bar{F}_{\rho}$ normalizes $T$.

Let $t \in T$. Then

$$
t=\mathcal{V}(g)=\prod_{i=1}^{m} \lambda_{v_{i}}\left(g_{i}\right) \quad \text { for some } g \in\left(S^{1}\right)^{m},
$$

where (2.11) is used for the latter identity. Using (7.13) together with the definition of $\mathcal{D}$ (see also (7.1)), (7.12), (7.11) and (7.6), we have

$$
\begin{aligned}
\left(\mathcal{D}\left(\bar{F}_{\rho}\right)\right)(t) & =\bar{F}_{\rho} \circ t \circ \bar{F}_{\rho}^{-1}=\bar{F}_{\rho} \circ \mathcal{V}(g) \circ \bar{F}_{\rho}^{-1}=\mathcal{V}(\phi(g)) \\
& =\mathcal{V}\left(g_{\sigma(1)}, \ldots, g_{\sigma(m)}\right)=\prod_{i=1}^{m} \lambda_{v_{i}}\left(g_{\sigma(i)}\right)=\prod_{i=1}^{m} \lambda_{\rho_{*}\left(v_{\sigma}(i)\right)}\left(g_{\sigma(i)}\right) \\
& =\rho\left(\prod_{i=1}^{m} \lambda_{v_{\sigma(i)}}\left(g_{\sigma(i)}\right)\right)=\rho\left(\prod_{i=1}^{m} \lambda_{v_{i}}\left(g_{i}\right)\right)=\rho(\mathcal{V}(g))=\rho(t)
\end{aligned}
$$

and this proves the latter statement in the lemma. 


\section{Maximal compact Lie subgroup of $\operatorname{Symp}(M, \omega)$}

The purpose of this section is to prove the following.

Theorem 8.1. If a compact Lie subgroup $G$ of $\operatorname{Symp}(M, \omega)$ containing the torus $T$ satisfies the following two conditions:

(1) $\Delta(G)=R(P)$, and

(2) the map $\mathcal{D}$ in (7.2) is surjective,

then $G$ is maximal among compact Lie subgroups of $\operatorname{Symp}(M, \omega)$ containing the torus T. Moreover, there is a compact Lie subgroup $G_{\max }$ of $\operatorname{Symp}(M, \omega)$, which satisfies conditions (1) and (2) above.

Proof. What we prove for the former part of the theorem is that if a compact Lie subgroup $H$ of $\operatorname{Symp}(M, \omega)$ contains the $G$ in the theorem, then $H=G$.

Since $G$ is a subgroup of $H$, the root system $\Delta(G)$ of $G$ is a subsystem of the root system $\Delta(H)$ of $H$. On the other hand, since $H$ is a compact Lie subgroup of $\operatorname{Symp}(M, \omega)$ containing $T, \Delta(H)$ is a subsystem of $R(P)$. Therefore, it follows from condition (1) in the theorem that $\Delta(G)=\Delta(H)$ and this shows that $G^{0}=H^{0}$ where the superscript 0 denotes the identity components as before.

Since $G$ is a subgroup of $H, N_{G}(T)$ is a subgroup of $N_{H}(T)$. It follows from Lemma 7.1 and the condition (2) in the theorem that $N_{G}(T)=N_{H}(T)$ and this together with the isomorphism (7.3) for $G$ and $H$ implies $G=H$ because $G^{0}=H^{0}$.

The proof of the latter part of the theorem is as follows. Let $\tilde{G}_{\max }$ be the subgroup of $U(m)$ generated by $\tilde{G}$ in Proposition 6.1 and $F_{\rho}$ 's in (7.10) (regarded as elements of $\mathrm{U}(m)$ ) for $\rho \in \operatorname{Aut}(P)$. The identity component of $\tilde{G}_{\max }$ is $\tilde{G}$. The action of $\tilde{G}_{\max }$ on $\mathbb{C}^{m}$ leaves $\mathcal{Z}_{P}$ invariant and induces an effective action of $G_{\max }:=\tilde{G}_{\max } / \operatorname{Ker} \mathcal{V}$ on $M$ preserving $\omega$. The group $G_{\max }$ contains the torus $T$ and satisfies the two conditions in the theorem by Proposition 6.1 and Lemma 7.4.

\section{Note added in proof.}

After the paper was accepted for publication, the author was informed from Professor D. McDuff that Proposition 5.5 in her paper with S. Tolman "Polytopes with mass linear functions. I, Int. Math. Res. Not. IMRN 2010, no. 8, 1506-1574" is essentially the same as our Theorem 1.1 concerning the identity component of the compact Lie subgroup $G$ or $G_{\max }$.

\section{References}

[1] F. Bosio and L. Meersseman, Real quadrics in $\mathbb{C}^{n}$, complex manifolds and convex polytopes, Acta Math. 197 (2006), 53-127. 
[2] V. M. Buchstaber and T. E. Panov, Torus actions and their applications in topology and combinatorics, University Lecture, vol. 24, Amer. Math. Soc., Providence, RI, 2002.

[3] D. Cox, The homogeneous coordinate ring of a toric variety, J. Algebraic Geom. 4 (1995), 17-50.

[4] T. Delzant, Hamiltoniens périodiques et image convex de l'application moment, Bull. Soc. Math. France 116 (1988), 315-339.

[5] M. Demazure, Sous-groupes algébriques de rang maximum du groupe de Cremona, Ann. Sci. École Norm. Sup., (4) 3 (1970), 507-588.

[6] V. Guillemin, Moment maps and combinatorial invariants of Hamiltonian $T^{n}$-spaces, Prog. in Math., vol. 122, Birkhäuser, 1994.

[7] A. Hattori and M. Masuda, Theory of multi-fans, Osaka J. Math. 40 (2003), 1-68.

[8] Y. Karshon, L. Kessler and M. Pinsonnault, A compact symplectic four-manifold admits only finitely many inequivalent toric actions, J. Symp. Geom. 5 (2007), 139166.

[9] J. E. Humphreys, Introduction to Lie algebras and representation theory, Graduate Texts in Math, vol. 9, Springer-Verlag, Berlin, 1970.

[10] M. Masuda. Unitary toric manifolds, multi-fans and equivariant index, Tohoku Math. J. 51 (1999), 237-265.

[11] T. Oda, Convex bodies and algebraic geometry. An introduction to the theory of toric varieties, Ergeb. Math. Grenzgeb. (3), vol. 15, Springer-Verlag, Berlin, 1988.

[12] M. Wiemeler, Torus manifolds with non-abelian symmetries, arXiv:0911.4936.

Department of Mathematics

OSAKA City UnIVERSITY

SUMIYOSHI-KU

OSAKA $558-8585$

JAPAN

E-mail address: masuda@sci.osaka-cu.ac.jp

Received 08/06/2009, accepted 03/03/2010

I would like to thank Nigel Ray for his invitation to University of Manchester in summer of 2008 and stimulating discussions. This work is an outcome of a project with him. I also would like to thank Michael Wiemeler for explaining his work [12], which motivated the introduction of the root system $R(P)$. Finally, I would like to thank Megumi Harada and Shintaro Kuroki for their helpful comments on an earlier version of the paper. The author was partially supported by Grant-in-Aid for Scientific Research 19204007. 